\title{
Pseudo-carcinomatous invasion in adenomatous polyps of the colon and rectum
}

\author{
T. MUTO, H. J. R. BUSSEY, AND B. C. MORSON
}

From St Mark's Hospital, London

SYNOPSIS The histology of pseudo-carcinomatous invasion in adenomatous polyps of the colon and rectum is described and the appearances are contrasted with those seen in malignant polyps.

The recognition of pseudo-carcinomatous invasion is important in the differential diagnosis of benign and malignant polyps of the large bowel. Failure to distinguish it from carcinoma may lead to wrong treatment and false reports of the incidence and prognosis of cancer of the colon and rectum.

The evidence suggests that pseudo-carcinomatous invasion may be the result of repeated twisting of the stalk of a polyp which causes haemorrhage and this facilitates the passage of non-malignant adenomatous epithelium through the muscularis mucosae.

During the course of an investigation into the histology and prognosis of a large number of adenomatous polyps of the colon and rectum a group was identified in which epithelial tubules were found in the submucosal layer in continuity with the neoplastic tissue in the head of the polyp. The histology was different from invasion by carcinoma across the line of the muscularis mucosae into the submucosa, as seen in malignant polyps. The object of this paper is to describe these histological differences and to provide evidence to show that they are not carcinomatous and are most probably the result of misplacement of adenomatous epithelium due to twisting of the stalk of the polyp. It is clearly important from the point of view of surgical treatment of polypoid tumours of the colon and rectum that pseudocarcinomatous invasion should be distinguished from cancer.

\section{Material and Methods}

Fifty-six polyps from 54 patients showed the histological criteria for pseudo-carcinomatous invasion. They were extracted from a series of 2341 polyps removed from 1586 patients seen at St Mark's Hospital during the 12-year period 1957-1968. In this same period 110 polyps (both adenomatous and villous tumours) were examined in which there was unequivocal evidence of invasive carcinoma into the submucosal layer only. From these figures it will be realized that pseudo-carcinomatous invasion is quite common.

Received for publication 26 October 1972.
All the polyps were embedded in paraffin wax in such a way as to allow for correct microanatomical orientation of the tumour, the submucosal layer and its stalk. In most cases multiple sections through the whole polyp and its stalk were available for study. The sections were stained with haematoxylin and eosin, Masson's trichrome, Perl's Prussian blue, and alcian blue.

\section{Histology (Figs. 1-4)}

The most essential feature for the diagnosis of malignancy in adenomatous polyps of the colon and rectum is the spread of adenocarcinoma across the line of the muscularis mucosae (Grinnell and Lane, 1958; Morson, 1966). Whatever the terminology given to neoplastic changes superficial to this line (Morson and Bussey, 1970), once cancer cells have reached the submucosal layer they undoubtedly have the potential for metastasis as well as further spread in continuity. Examples of 'carcinoma' superficial to the line of the muscularis mucosae from which metastasis has taken place are very few and are doubtfully authentic (Palacios and Wellmann, 1966). All the polyps showing pseudo-carcinomatous invasion were typical adenomas in which similar adenomatous tissue was found in the submucosa beneath the muscularis mucosae. The appearance was significantly different from invasive carcinoma.

In the polyps with pseudo-carcinomatous invasion gland-like structures in the submucosa were lined by epithelium which showed the same degree of dysplasia as in the head of the polyp and histological 


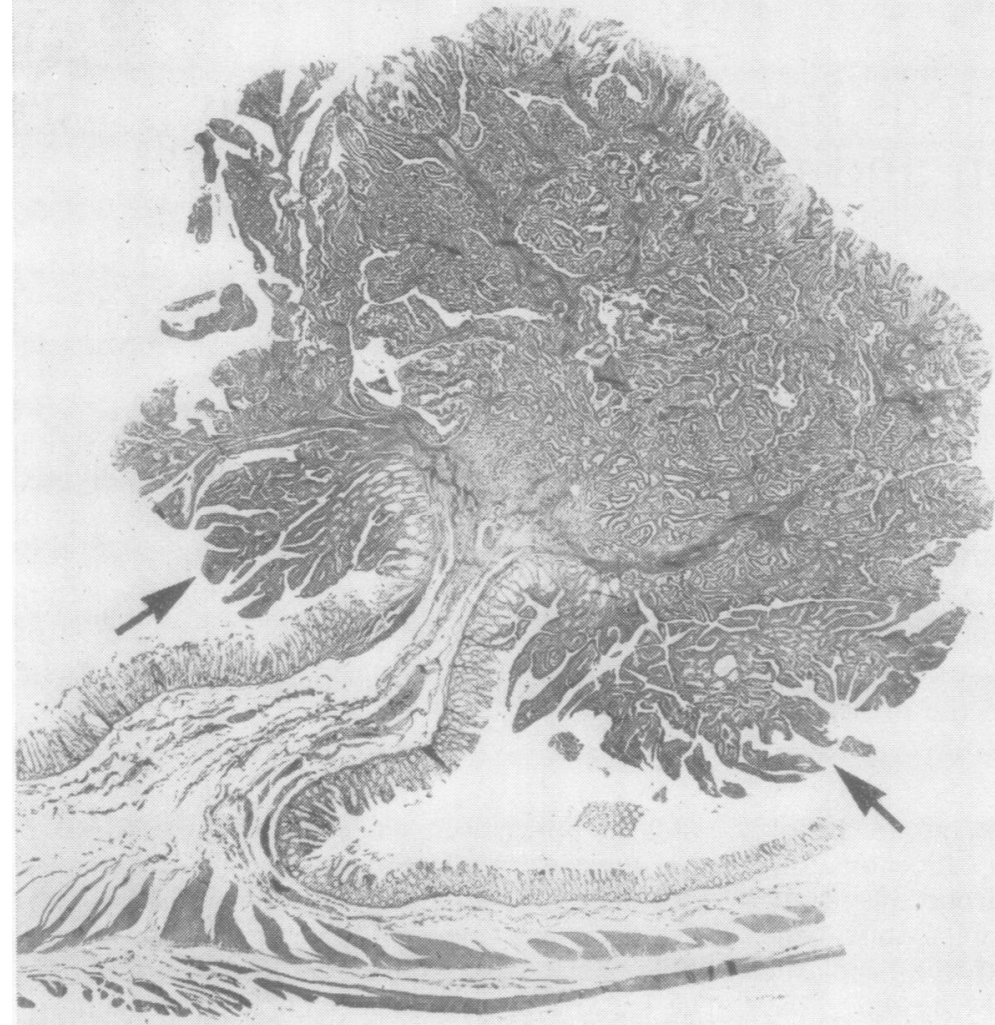

Fig. 1a Typical malignant polyp. Much of the tumour is an adenocarcinoma invading the stalk of the polyp, but there is residual benign adenomatous tissue at the margins (arrowed). Compare with Fig. $2 a . \mathrm{H} \& \mathrm{E} \times 10$

Fig. 1b High-power view of the invading edge of the carcinoma in Fig. la showing the desmoplastic reaction around the carcinomatous glands. Compare with Fig. 2c. $H \& E \because 100$

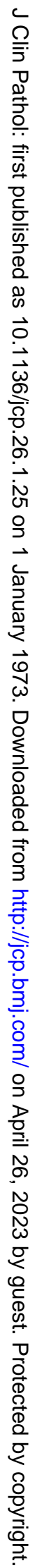

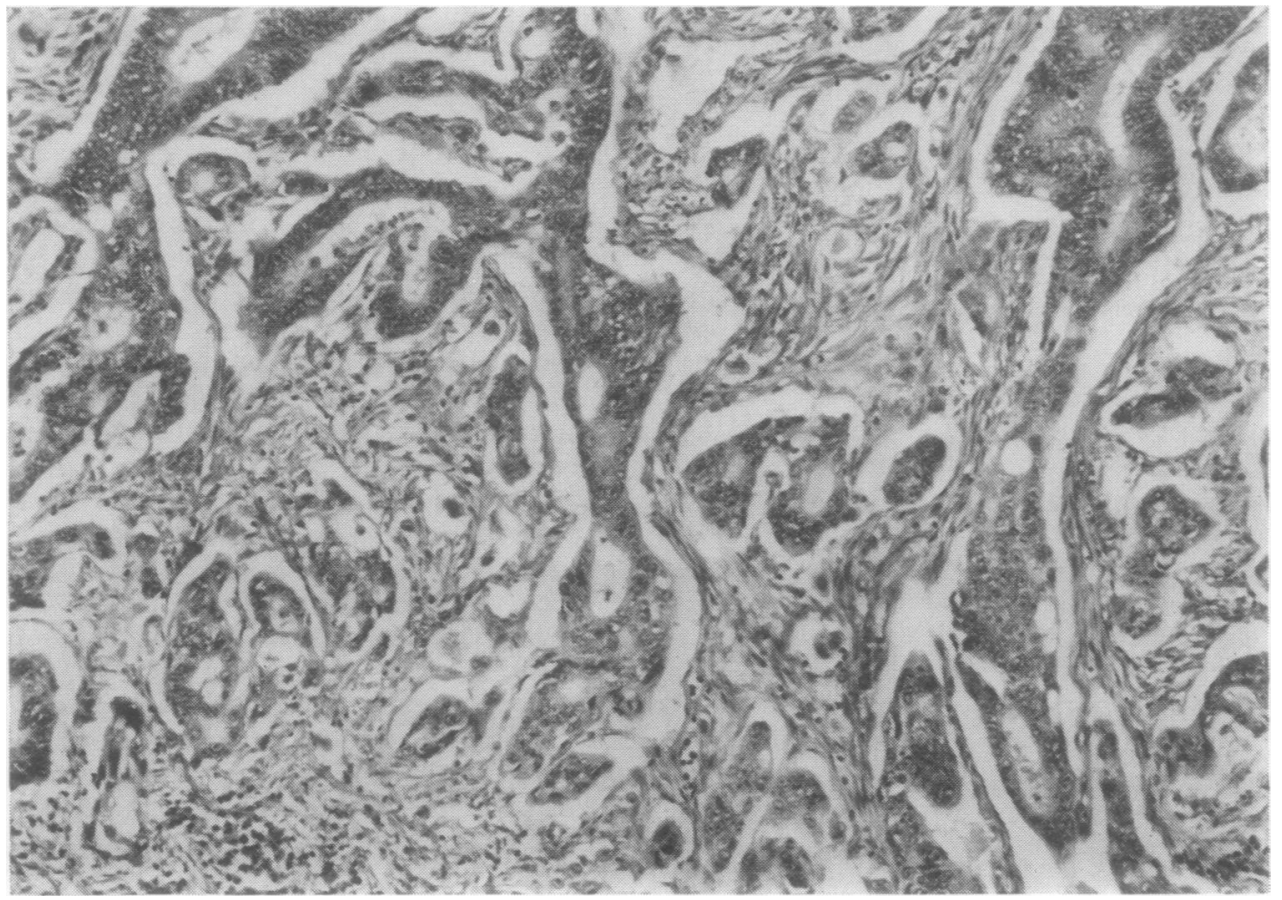



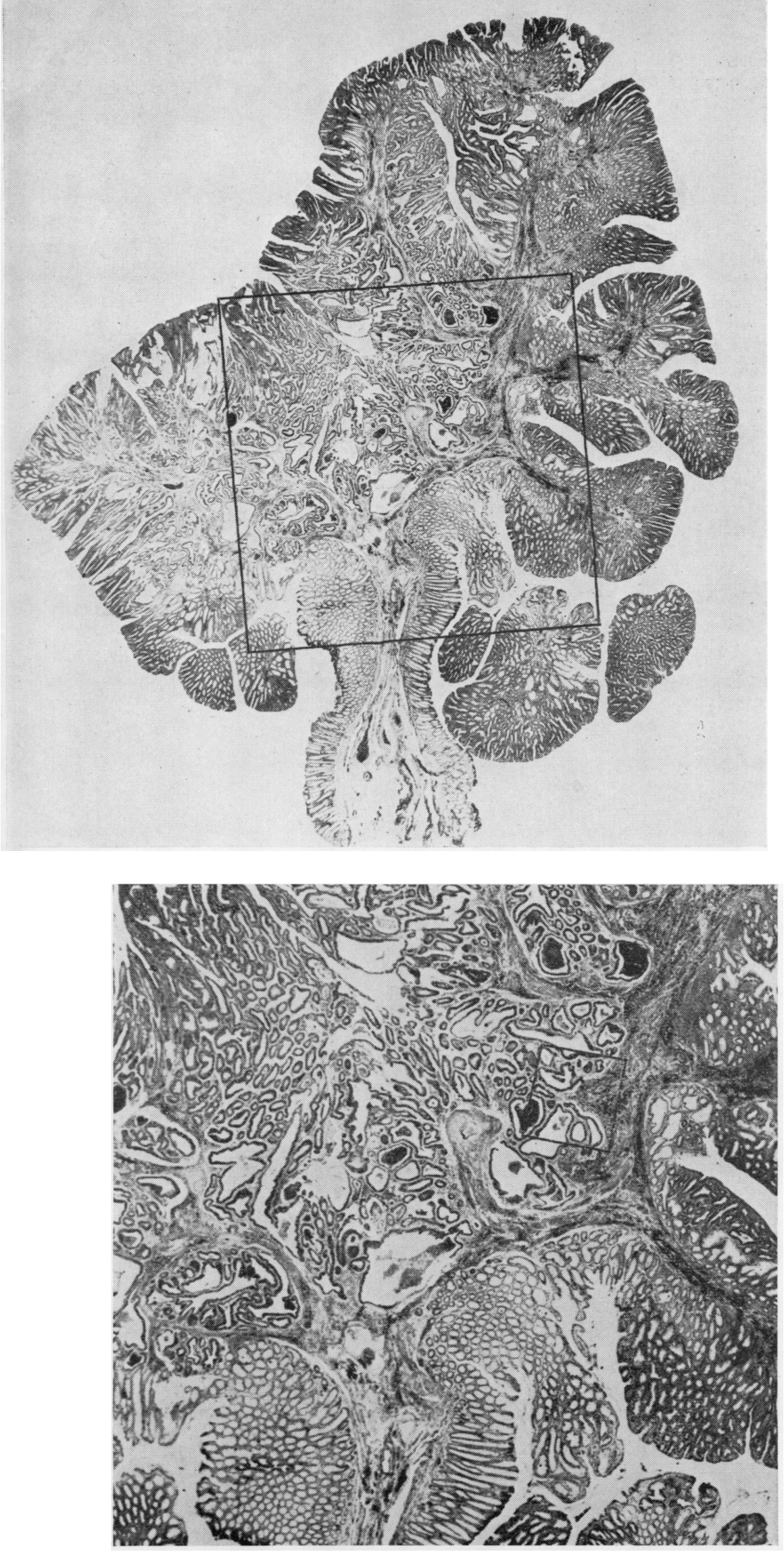

Fig. 2a Adenomatous polyp with pseudo-carcinomatous invasion of $\overrightarrow{0}$ the stalk. $H \& E \times 10$

Fig. 2b High-power view of inset $\dot{\text { on }}$ Fig. 2a. There are glandular elements in the submucosal layer, some of them showing cystic change, which are arranged in clumps. There is $N$ continuity with the adenomatous tissue in the head of the polyp and $N$ the clumps are surrounded by a N chronic inflammatory reaction containing haemosiderin pigment. $H \& E \times 15$ 


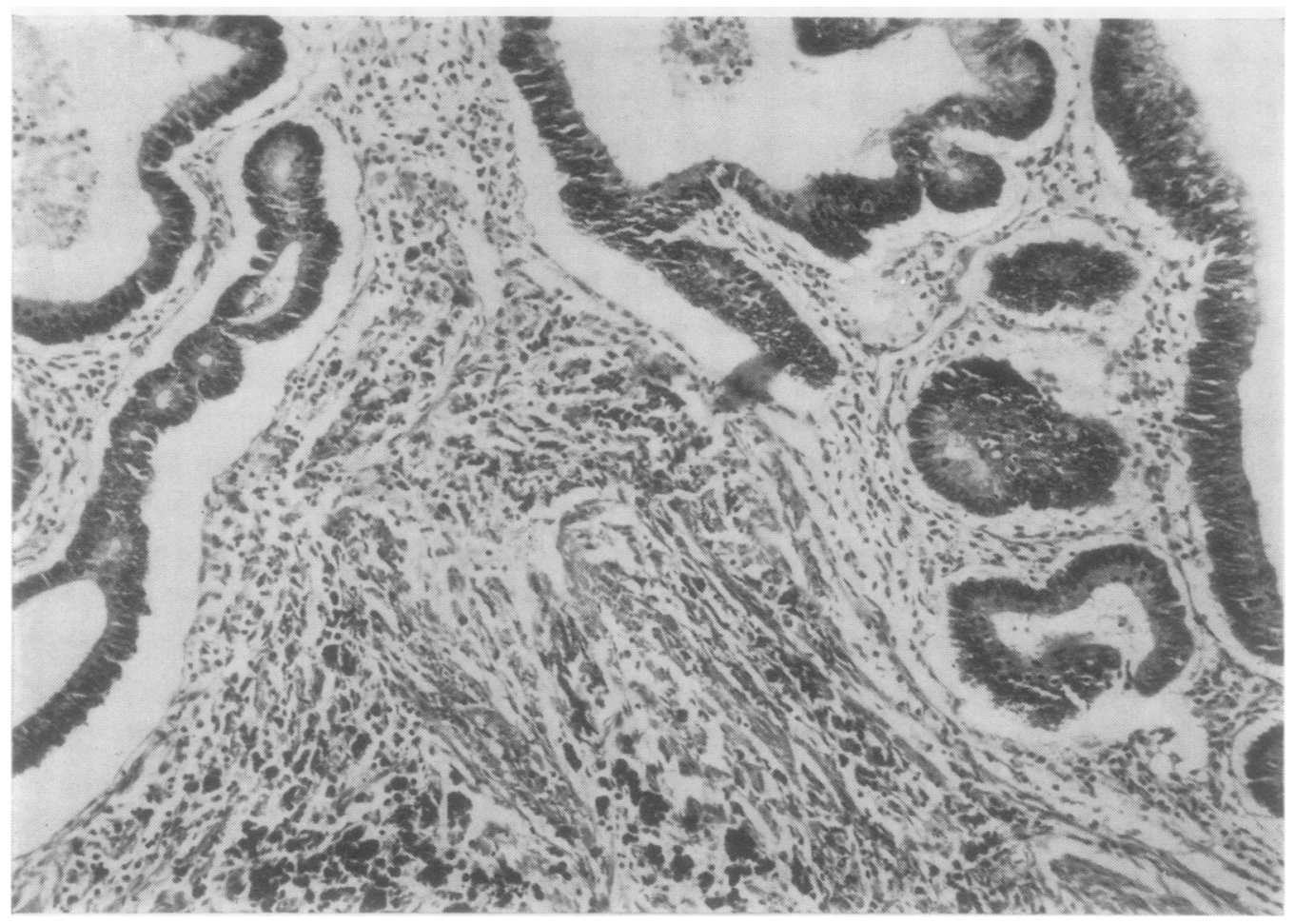

Fig. 2c High-power view of inset in Fig. $2 b$ to show glands lined by dysplastic epithelium lying in a connective tissue stroma identical with the lamina propria in adenomatous polyps. The inflammatory reaction and fibrosis at the margin of the clump of glands contains much black pigment which has the staining characteristics of haemosiderin. Compare with Fig. $1 b . H \& E \times 40$

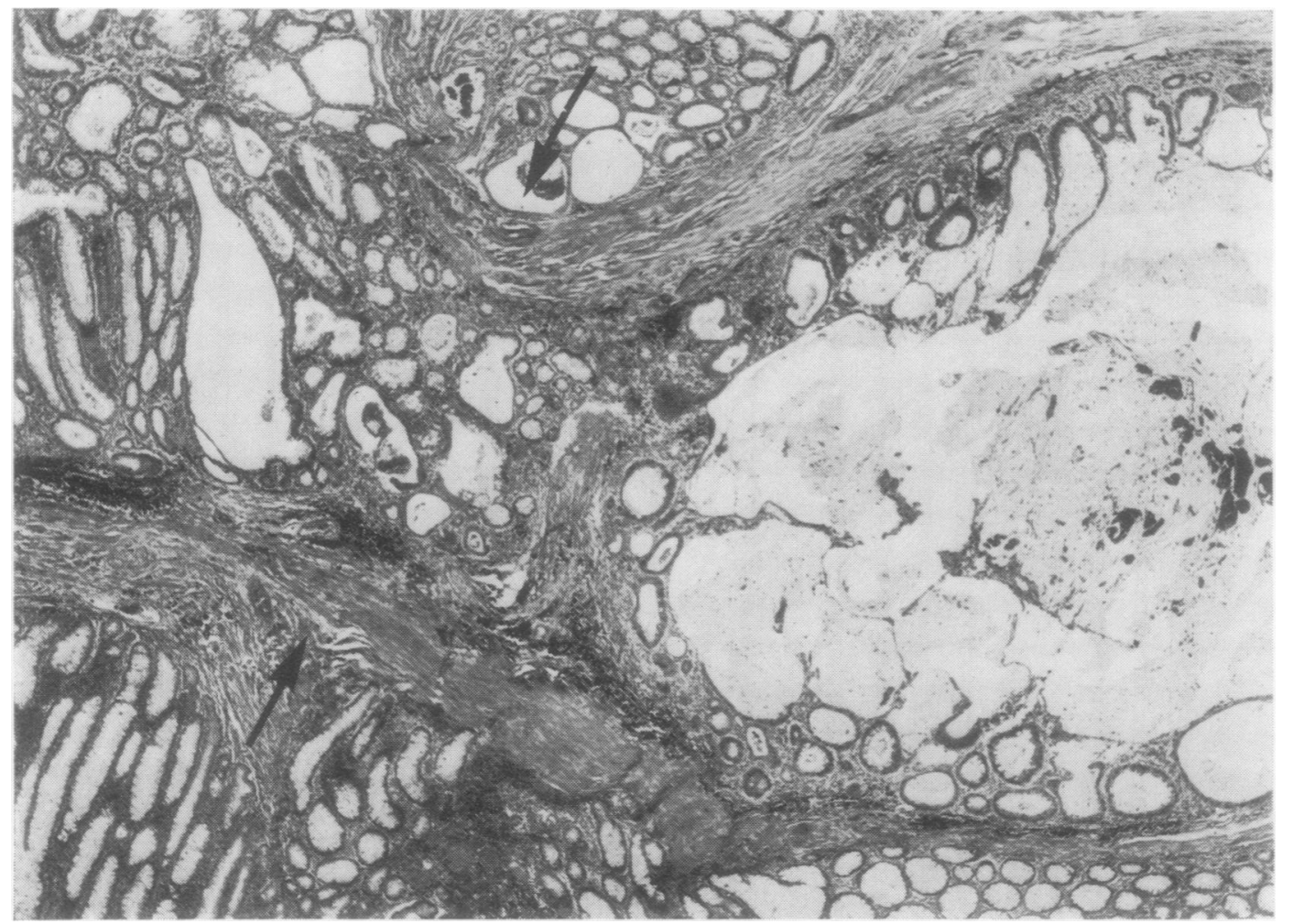

Fig. 3 Histology of pseudo-carcinomatous invasion. Adenomatous glands misplaced through the muscularis mucosae (arrowed) into the submucosa on the right carrying lamina propria with them. $H \& E \times 25$. 


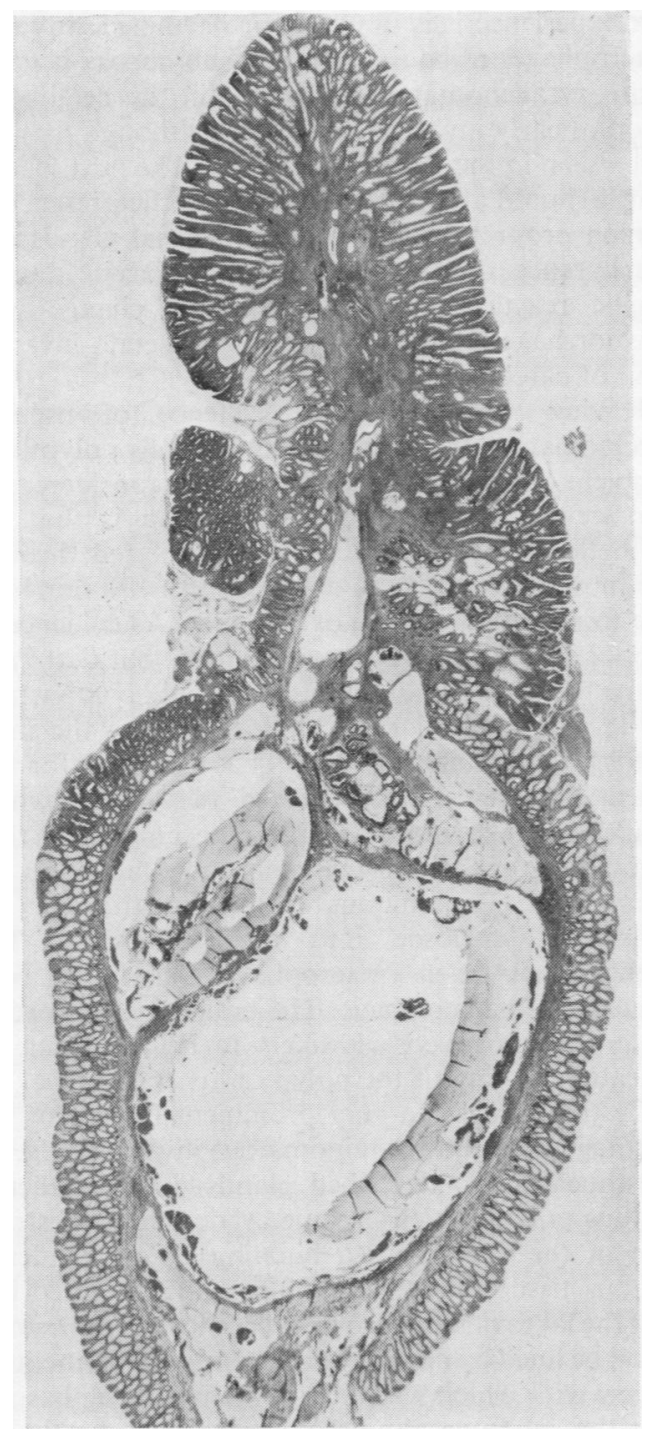

Fig. 4 Adenomatous polyp with pseudo-carcinomatous invasion of the stalk. The latter contains multiloculated cysts filled with mucin. $H \& E \times 10$.

continuity across the line of the muscularis mucosae could be established. The submucosal glands were, however, surrounded by lamina propria without any desmoplastic reaction to the epithelial cells which is usual in invasive carcinoma. Cystic change was very pronounced in many of the pseudo-carcinomatous glands, and this is unusual and less pronounced in true invasive carcinoma. Much retention of mucus within glands with atrophy of lining epithelium was a feature of some cases and the distinction from early mucinous or colloid carcinoma is thus particularly important. In pseudo-carcinomatous invasion the submucosal glandular tissue was well circumscribed without the characteristics of malignant infiltration.

In 48 out of 56 polyps showing pseudo-carcinomatous invasion there were deposits of pigment with the staining characteristics of haemosiderin around the submucosal glands. In some cases this was massive and a very obvious feature of the histology. The head of an adenomatous polyp in our experience not infrequently contains deposits of haemosiderin. but not in the submucosa of the stalk beneath. Recent haemorrhage into and around the gland-like structures in the submucosa was also quite a common feature of pseudo-carcinomatous polyps. It is presumably this which gives rise to accumulating deposits of haemosiderin. The gland-like structures in these cases were usually surrounded by a narrow rim of granulation tissue composed of collagen and inflammatory cells. It is in this tissue that the heaviest deposits of haemosiderin were found. This gave a clearly demarcated histological appearance in contrast to the infiltrating appearance of early invasive carcinoma.

Another prominent feature of some pseudocarcinomatous polyps was the marked branching and excess of muscularis mucosae. In many it appeared as if adenomatous tissue from the head of the polyp was being squeezed through gaps in the distorted and thickened muscle.

\section{Statistical Analysis}

\section{AGE AND SEX}

The average age of the patients with pseudocarcinomatous polyps was 60 years for men and 56 years for women, which is similar to the average age of ordinary adenomatous polyps, but slightly lower than for carcinoma of the colon and rectum (Morson and Bussey, 1970). Men were more commonly affected than women in a ratio of $3: 1$.

\section{SITE OF THE POLYPS}

The polyps were situated as follows and showed a striking preference for the sigmoid part of the large bowel:

\begin{tabular}{llllllllr}
\hline $\begin{array}{l}\text { Descending colon } \\
\text { Sigmoid colon }\end{array}$ &.. &.. &.. &.. &.. &.. &.. & 5 \\
Recto-sigmoid &.. &.. &.. &.. &.. &.. &.. & 36 \\
$\begin{array}{l}\text { Rectum } \\
\text { Total }\end{array}$ &.. &.. &.. &.. &.. &.. &.. & 7 \\
\hline
\end{tabular}


SIZE

Most of the polyps were much larger than average and measured as follows:

Under $1 \mathrm{~cm}$.

$1-2 \mathrm{~cm}$

$2-3 \mathrm{~cm}$

Over $3 \mathrm{~cm}$

LENGTH OF STALK

In five polyps the length of the stalk was not available. In three cases the polyp was sessile. In the remaining 48 polyps the stalk measured as follows:

Less than $0.5 \mathrm{~mm}$

$0.5-1.0 \mathrm{~cm}$

Over $1.0 \mathrm{~cm}$

Thus, most of the polyps showing pseudocarcinomatous invasion had exceptionally long stalks.

\section{PROGNOSIS}

In 20 out of 54 patients the pseudo-carcinomatous polyps were found in surgical specimens resected for an obvious cancer of the colon or rectum. In none of these was there any evidence of metastasis to regional lymph nodes from the pseudo-carcinomatous tumours.

In 34 patients the pseudo-carcinomatous polyps were single lesions treated either by resection or local excision. The follow up in the group ranges from one year to over five years in five of the patients. There has been no evidence of recurrence or metastasis.

\section{Discussion}

The recognition of pseudo-carcinomatous invasion in adenomatous polyps and its distinction from invasive cancer clearly has considerable importance in surgical treatment. Moreover, it may be sufficiently common to raise doubts about the accuracy of published figures for the incidence of malignant polyps and early cancer of the colon and rectum. There is no indication from a study of the literature that pathologists are fully aware of the problem. At least two examples of what we would regard as pseudo-carcinomatous invasion have been reported as adenocarcinoma (Hultborn, 1954; Veidenheimer, Connolly, and Legg, 1970).

The presence of haemosiderin deposits in the stalk of a 'malignant polyp' should be the first indication that the presence of neoplastic glands in the submucosa may represent a process of pseudoinvasion rather than carcinomatous infiltration. In our experience it is unusual for haemosiderin-laden $\stackrel{\frac{0}{\vec{\sigma}}}{\underline{0}}$ macrophages to be found in the submucosa beneath ordinary adenomatous polyps or in the neighbour- $\frac{\vec{s}}{9}$ hood of early invasive carcinomas although they are often seen in the head of the polyp. The next feature which should alert the pathologist is the presence of $\frac{\sigma}{7}$ lamina propria around the submucosal glands and $\stackrel{\complement}{\complement}$ the absence of any immediately adjacent desmo- $\cong$ plastic reaction. Last, much cystic change with $\vec{\circ}$ haemorrhage is more suggestive of pseudo-invasion than of carcinoma.

Having recognized the existence of pseudo- $\stackrel{\mathscr{S}}{\mathscr{S}}$ carcinomatous invasion in adenomatous polyps, the $\frac{8}{8}$ problem of its pathogenesis has to be considered. In our view the most likely explanation is that the epithelium in the submucosa is misplaced through ơ the muscularis mucosae as a result of haemorrhage 0 due to repeated twisting of the stalk. As evidence in support of this theory we would point out that most $c$ of the polyps were found in the sigmoid colon which is the part of the large intestine showing the most powerful muscular activity. This accounts for the unusually long stalks, repeated twisting of which $\vec{\theta}$ would cause haemorrhage into the polyp and this, $\omega$ together with secondary inflammation, facilitates the passage of adenomatous epithelium through the muscularis mucosae into the submucosa. The haemosiderin-laden macrophages are the consequence of haemorrhage. The branching and excess of muscularis mucosa is likely to be the result of repeated twisting of the polyp on its stalk.

Cystic change is a very prominent feature of the histology of pseudo-carcinomatous invasion (Fig. 4). Westhues (1934) described glands deep in adenomatous polyps which became cystic due to obstruction of the ducts with a bursting effect of adenomatous tissue into the submucosa. He pointed out that the lakes of mucus so produced ('Schleimstrasse') could be lined by normal or adenomatous epithelium, a view with which we concur. Westhues emphasized that the submucosal epithelium in his cases should not be regarded as carcinomatous.

Follow up of our patients has, so far, shown no $N$ sign of recurrence or metastasis which supports the concept that the appearances are due to pseudo- $N$ carcinomatous invasion. All the evidence suggests $\mathbb{W}$ that this is a benign condition and that local excision should be sufficient treatment.

There are reports in the literature of 'localized $\frac{D}{\mathscr{D}}$ colitis cystica profunda' and 'hamartomatous $\stackrel{?}{+}$ inverted polyps' of the rectum in which glandular 0 epithelium is found in the submucosa but is covered by non-neoplastic mucous membrane (Allen, 1966; Epstein, Ascari, Ablow, Seamen, and Lattes, 1966; 용 Wayte and Helwig, 1967). This condition is quite different from "colitis cystica profunda in an 8 
adenoma' (Fechner, 1967; Clark and Muldoon, 1970) which seems to be an expression used to describe what we here call 'pseudo-carcinomatous invasion in an adenomatous polyp'.

This investigation received financial support from the World Health Organization, the Cancer Research Campaign, and the St Mark's Research Foundation.

We are grateful to Mr Lloyd Soodeen, AIMLT, for technical assistance; to Mr Norman Mackie, Senior Photographer at St Mark's Hospital, for the microphotography of Figs. 1 and 2, and to $\mathbf{M r}$ Ronald Barnett, and Professor W. St. C. Symmers, Department of Medical Illustration, Charing Cross Hospital Medical School, for Figures 3 and 4.

References

Allen, M. S., Jr. (1966). Hamartomatous inverted polyps of the rectum. Cancer (Philad.), 19, 257-265.

Clark, J. F., and Muldoon, J. P. (1970). Colitis cystica profunda in an adenoma (adenomatous polyp). Dis. Colon Rectum, $13387-$ 389.

Epstein, S. E., Ascari, W. Q., Ablow, R. C., Seaman, W. B., and Lattes, R. (1966). Colitis cystica profunda. Amer. J. clin. Path. 45, $186-201$.

Fechner, R. E. (1967). Polyp of the colon possessing features of colitis cystica profunda. Dis. Colon. Rectum, 10, 359-367.

Grinnell, R. S., and Lane, N. (1958). Benign and malignant adenomatous polyps and papillary adenomas of the colon and rectum: an analysis of 1856 tumours in 1335 patients. Surg. Gynec. Obstet., 106, 519-538.

Hultborn, K. A. (1954). The causal relationship between benign epithelial tumors and adenocarcinoma of the colon and rectum. Acta radiol. (Stockh.), Suppl. 113.

Morson, B. C. (1966). Factors influencing the prognosis of early cancer of the rectum. Proc. roy. Soc. Med., 59, 607-608.

Morson, B. C., and Bussey, H. J. R. (1970). Predisposing causes of intestinal cancer. Curr. Probl. Surg., $1-46$.

Palacios, R. L., and Wellmann, K. F. (1966). Adenomatous polyp of colon with adenocarcinoma and pulmonary metastases. Gastroenterology, 51, 82-86.

Veidenheimer, M. C., Connolly, J. M., and Legg, M. A. (1970). Carcinoma in colonic and rectal polyps. Dis. Colon Rectum, 13, 194-200.

Wayte, D. M., and Helwig, E. B. (1967). Colitis cystica profunda. Amer. J. clin. Path., 48, 159-169.

Westhues, H. (1934). Die pathologisch-anatomischen Grundlagen der Chirurgie des Rektumkarzinoms. Thieme, Leipzig. 